\title{
TU/e EmonOWEN

\section{Rotation of a sphere in a viscoelastic liquid subjected to flow. Part I: Simulation results}

\section{Citation for published version (APA):}

D Avino, G., Hulsen, M. A., Snijkers, F., Vermant, J., Greco, F., \& Maffettone, P. L. (2008). Rotation of a sphere in a viscoelastic liquid subjected to flow. Part I: Simulation results. Journal of Rheology, 52(6), 1331-1346. https://doi.org/10.1122/1.2998219

DOI:

$10.1122 / 1.2998219$

Document status and date:

Published: 01/01/2008

\section{Document Version:}

Publisher's PDF, also known as Version of Record (includes final page, issue and volume numbers)

\section{Please check the document version of this publication:}

- A submitted manuscript is the version of the article upon submission and before peer-review. There can be important differences between the submitted version and the official published version of record. People interested in the research are advised to contact the author for the final version of the publication, or visit the $\mathrm{DOI}$ to the publisher's website.

- The final author version and the galley proof are versions of the publication after peer review.

- The final published version features the final layout of the paper including the volume, issue and page numbers.

Link to publication

\section{General rights}

Copyright and moral rights for the publications made accessible in the public portal are retained by the authors and/or other copyright owners and it is a condition of accessing publications that users recognise and abide by the legal requirements associated with these rights.

- Users may download and print one copy of any publication from the public portal for the purpose of private study or research.

- You may not further distribute the material or use it for any profit-making activity or commercial gain

- You may freely distribute the URL identifying the publication in the public portal.

If the publication is distributed under the terms of Article 25fa of the Dutch Copyright Act, indicated by the "Taverne" license above, please follow below link for the End User Agreement:

www.tue.nl/taverne

Take down policy

If you believe that this document breaches copyright please contact us at:

openaccess@tue.nl

providing details and we will investigate your claim. 


\title{
Rotation of a sphere in a viscoelastic liquid subjected to shear flow. Part I: Simulation results
}

\author{
Gaetano D’Avino ${ }^{\text {a) }}$ \\ Dipartimento di Ingegneria Chimica, Università degli Studi di Napoli \\ Federico II, Piazzale Tecchio 80, 80125 Napoli, Italia \\ Martien A. Hulsen \\ Department of Mechanical Engineering, Eindhoven University of Technology, \\ PO Box 513, 5600 MB Eindhoven, The Netherlands \\ Frank Snijkers and Jan Vermant \\ Department of Chemical Engineering, K.U. Leuven, W. de Croylaan, \\ B-3001 Leuven, Belgium \\ Francesco Greco \\ Istituto di Ricerche sulla Combustione, IRC, CNR, Piazzale Tecchio 80, \\ 80125 Napoli, Italia \\ Pier Luca Maffettone \\ Dipartimento di Ingegneria Chimica, Università degli Studi di Napoli, \\ Federico II, Piazzale Tecchio 80, 80125 Napoli, Italia
}

(Received 27 November 2007; final revision received 17 September 2008)

\section{Synopsis}

In inertialess suspensions of rigid particles, the rotational motion of each particle is governed by the so-called freely rotating condition, whereby the total torque acting on the particle must be zero. In this work, we study the effect of viscoelasticity of the suspending liquid on the rotation period of a sphere by means of three-dimensional finite element simulations, for conditions corresponding to a macroscopic shear flow. The simulation results capture the slowing down of the rotation, relative to the Newtonian case, which was recently observed in experiments. It is shown that such a phenomenon depends on the specific constitutive equation adopted for the viscoelastic liquid. Analysis of transients shows a clear correlation between rotation rate and the development of first normal stress difference. (C) 2008 The Society of Rheology. [DOI: 10.1122/1.2998219]

\section{INTRODUCTION}

In dilute suspensions of buoyancy-free rigid non-Brownian particles, externally imposed flow fields determine forces and torques on the particles, resulting in their trans-

\footnotetext{
a) Author to whom correspondence should be addressed; electronic mail: gadavino@unina.it
} 
lations and rotations. Diluteness of the suspension implies that the particles do not feel each other. Hence, the motion of a particle only depends on the particle characteristics, e.g., shape, surface properties, boundary conditions, and on the rheological features of the suspending liquid. We consider here the simple case of inertialess, buoyancy free, spherical particles, for which no-slip conditions hold.

The problem of the dynamics of a single sphere immersed in a linear flow field imposed at infinity, in the absence of both fluid and particle inertia, was first addressed by Einstein (1906) for the case of a Newtonian suspending medium. Under steady shear flow, the sphere translates in the flow direction, while rotating around the vorticity axis. Thus, in a frame translating with the sphere center, the sphere just rotates in time with a constant angular velocity $\omega$. Einstein (1906) demonstrated that, under no-slip boundary conditions at the particle surface, the rotation rate $\omega=\dot{\gamma} / 2, \dot{\gamma}$ being the externally imposed shear rate. This simple result stems from a torque balance at the sphere surface, whereby the torque on the sphere is only due to the flow field (the so called torque-free condition).

In the case of a viscoelastic suspending liquid, the presently known analytical results were derived in the limit of a second order fluid (SOF) [Truesdell and Noll (1965)], i.e., the most general viscoelastic constitutive equation for the stress tensor, quadratic in the velocity gradient, describing essentially any viscoelastic liquid in the limit of slow and slowly varying flows. As first reported by Brunn (1976), it turns out that the angular velocity $\omega$ remains the same as in the Newtonian case. In experiments by Mason and co-workers [Gauthier et al. (1971)] on the rotational motion of single rigid particles in Couette flow of a shear thinning and a viscoelastic liquid, the rate of rotation of isolated particles was found to be identical to that observed in Newtonian fluids. Since the experiments were limited to slow flows, agreement with theoretical predictions for SOF was, in fact, expected.

In a recent paper Astruc et al. (2003) experimentally analyzed several suspensions, of both nearly spherical particles and agglomerates in viscoelastic liquids during steady state shear flow, abandoning the slow flow limit. Although the data are strongly scattered, a clear trend is identified, namely, particle rotation is observed to slow down as the shear rate increases. A similar slowing down in angular velocity is also found in recent numerical simulations by Hwang et al. (2004), for a two-dimensional (2D) suspension of disks in an Oldroyd-B liquid. We are not aware of any prediction for the three-dimensional (3D) case.

This paper is part of a joint effort to clarify the effects of a viscoelastic suspending liquid on the single sphere motion, as a first step towards the characterization of the rheological behavior of a dilute suspension. The problem will be quantitatively analyzed through novel 3D numerical simulations and newly designed experiments with model viscoelastic liquids. In the present paper, we present $3 \mathrm{D}$ simulation results on the single sphere shear problem, for various models of non-Newtonian suspending liquids. The effect of viscoelasticity on the angular velocity of the rotating sphere is examined for Deborah number up to 2.5 , hence, well beyond the slow flow limit. The constitutive equations are selected so as to evaluate the relative weight of shear thinning and normal stresses on the relative slowing down of the angular velocity. Transients will also be discussed.

\section{THE SINGLE SPHERE PROBLEM}

The hydrodynamic problem of a single sphere in an externally imposed flow field, under isothermal conditions, requires the solution of the continuity (mass balance) and 
momentum balance equations. As usual under Stokes flow conditions, fluid incompressibility, negligible fluid and particle inertia, and buoyancy free conditions are also assumed. The balance equations then read

$$
\begin{gathered}
\boldsymbol{\nabla} \cdot \mathbf{v}=0, \\
-\boldsymbol{\nabla} p+\boldsymbol{\nabla} \cdot \boldsymbol{\tau}=\mathbf{0},
\end{gathered}
$$

where $p$ is the pressure, $\mathbf{v}$ is the velocity, and $\boldsymbol{\tau}$ is the constitutive extra stress tensor.

Boundary conditions are assigned at infinity and on the sphere surface. The far-field condition corresponds to the undisturbed shear flow

$$
\begin{gathered}
p \rightarrow p^{\infty}, \\
\mathbf{v} \rightarrow \boldsymbol{\nabla} \mathbf{v}^{\infty} \cdot \mathbf{r},
\end{gathered}
$$

with $p^{\infty}$ as a constant pressure, $\nabla \mathbf{v}^{\infty}$ as the imposed velocity gradient, and $\mathbf{r}$ as the position vector relative to the sphere center. In the present work, the imposed velocity field at infinity is simple shear flow. Using Cartesian coordinates, $x$ corresponds to the flow direction, $y$ corresponds to the velocity gradient direction, and $z$ is the vorticity direction. Thus, at infinity, the imposed velocity gradient is $\boldsymbol{\nabla} \mathbf{v}_{x y}^{\infty}=\dot{\gamma}$, the other components being nil. No slip boundary conditions are imposed at the sphere surface $r=R$. We also assume, as mentioned in Sec. I, that the sphere is torque-free, or "freely rotating" [Brunn (1976); Leal (1979)], i.e., its rotation is only due to the motion of the surrounding fluid. Thus, the torque-free boundary condition at $r=R$ is

$$
\int_{A_{S}} \mathbf{r} \times \mathbf{T} \cdot \mathbf{n} d A=\mathbf{0},
$$

where $A_{\mathrm{s}}$ is the sphere surface, $\mathbf{n}$ is the normal at the sphere surface, the tensor $\mathbf{T}$ is $\mathbf{T}$ $=-p \mathbf{I}+\boldsymbol{\tau}$, and the integral of the local torque $\mathbf{r} \times \mathbf{T} \cdot \mathbf{n} d A$ spans the sphere surface.

Due to the symmetry of the imposed shearing flow, only the vorticity component in the $z$ direction is relevant in Eq. (3), the other two components being identically zero. In fact, the velocity at the sphere surface is known, and can be written as

$$
\mathbf{v}(t, R)=\omega(t) \boldsymbol{e}_{z} \times \mathbf{R},
$$

$\boldsymbol{e}_{\mathrm{z}}$ being the unit vector along $z$. The time-dependent velocity $\mathbf{v}(t, R)$ in Eq. (4) is at any point on the sphere surface the tangential velocity of that point. Hence, $\mathbf{v}(t, R)$ is everywhere tangential to the surface, and parallel to the shear plane $x y$. The unknown angular velocity $\omega(t)$ in Eq. (4) can then be determined through the $z$ component of the torquefree condition, Eq. (3).

\section{MATERIALS}

The aim of this paper is to investigate how the viscoelastic properties of the suspending liquid affect the rotation of a suspended sphere in shear flow. Therefore, our choice of constitutive equations [see, e.g., Larson (1988)] for the extra stress tensor $\boldsymbol{\tau}$ [see Eq. (1)] is so made as to highlight viscoelastic effects separately, i.e., viscosity thinning, and first and second normal stress differences, with or without thinning, are considered one at a time.

The effect of shear thinning is considered first, through the purely viscous BirdCarreau constitutive equation 


$$
\boldsymbol{\tau}=2 \frac{\eta_{0}}{\left(1+2 \lambda^{2} \mathbf{D}: \mathbf{D}\right)^{m}} \mathbf{D},
$$

where $\mathbf{D}$ is the rate-of-deformation tensor, $\eta_{0}$ is the zero shear viscosity, $\lambda$ is a characteristic time, and $m$ fixes the shear thinning slope. Note that, in the case of pure shear flow, it is $2 \mathbf{D}: \mathbf{D}=\dot{\gamma}^{2}$. Needless to say, the characteristic time in the Bird-Carreau equation does not relate to any time effect.

To describe time effects in viscoelastic liquids, the following three differential constitutive equations are hereafter considered:

$$
\begin{gathered}
\lambda \frac{\nabla}{\boldsymbol{\tau}}+\boldsymbol{\tau}=2 \eta_{0} \mathbf{D}, \\
\lambda \stackrel{\nabla}{\boldsymbol{\tau}}+\exp \left[\frac{\varepsilon \lambda}{\eta_{0}} \operatorname{tr}(\boldsymbol{\tau})\right] \boldsymbol{\tau}=2 \eta_{0} \mathbf{D}, \\
\lambda \frac{\nabla}{\boldsymbol{\tau}}+\frac{\alpha \lambda}{\eta_{0}} \boldsymbol{\tau} \cdot \boldsymbol{\tau}+\boldsymbol{\tau}=2 \eta_{0} \mathbf{D},
\end{gathered}
$$

where $\stackrel{\nabla}{\tau}$ indicates the upper convected (UC) time derivative of $\boldsymbol{\tau}$, and $\lambda$ is the relaxation time. Equation (6) is the UC Maxwell model, Eq. (7) is the Phan Thien-Tanner (PTT) model, and Eq. (8) is the Giesekus model. The latter two models contain each an additional parameter ( $\varepsilon$ and $\alpha$, respectively), and $\operatorname{tr}($.) represents the trace operator.

As it is well known, under steady state simple shear flow, the Maxwell model predicts a constant viscosity, a first normal stress difference $\left(N_{1}\right)$ quadratic in the shear rate, and no second normal stress difference $\left(N_{2}\right)$. In the PTT model, $N_{2}$ remains nil, whereas the viscosity and the first normal stress coefficient $\left(\Psi_{1}=N_{1} / \dot{\gamma}^{2}\right)$ are both dependent on the shear rate, becoming more pronouncedly shear thinning as $\varepsilon$ increases. Finally, in the Giesekus model, both normal stress differences are generated. The viscosity and both normal stress coefficients $\left(\Psi_{1}\right.$ and $\left.\Psi_{2}=-N_{2} / \dot{\gamma}^{2}\right)$ are all shear thinning. At any given shear rate, the parameter $\alpha$ modulates the extent of the thinning. As $\alpha$ increases, both the viscosity and $\Psi_{1}$ decrease, whereas $\Psi_{2}$ increases. Concerning the transient behavior of such fluids, it is worth recalling for later use that both the PTT and Giesekus models give rise to overshoots in the stress response at large shear rates, whereas the Maxwell model always predicts a monotonic behavior of the stress towards the steady state values.

The analysis is carried out by making the above equations dimensionless, using $\dot{\gamma}$ as the characteristic time scale, and $\eta_{0} \dot{\gamma}$ as the scale for the stress. Then, the Deborah number $\mathrm{De}=\lambda \dot{\gamma}$ appears in all the equations. The Deborah number will be used for the Bird-Carreau model as well, but in this case De is just a dimensionless shear rate. The Maxwell model is completely defined by De only, whereas in the other three models considered here one extra parameter has to be fixed $(m, \varepsilon$, or $\alpha)$.

\section{THE NUMERICAL PROCEDURES}

Once a constitutive equation is selected for the extra stress tensor $\boldsymbol{\tau}$, the single sphere problem for shear flow consists in the determination of the pressure and velocity fields, plus the angular velocity of the sphere $\omega$. The 3D flow problem was solved by the finite element method on a cubic cell, with $2 L$ as the length of the cubic side, containing a single sphere at the center of the cell. Due to the symmetry of the problem, only one quarter $(y>0$ and $z>0)$ of the full domain can be considered in order to optimize the computational effort. Indeed, the following conditions in the $x y$ and $x z$ plane hold: 


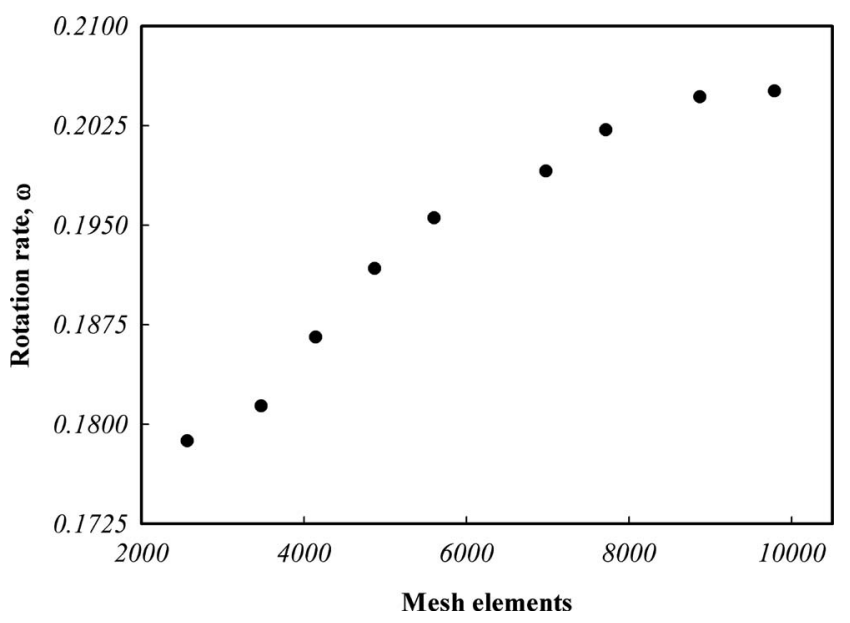

FIG. 1. Steady state rotation rate for different mesh resolutions. A Maxwell fluid is considered with De=2.5.

$$
\left.\begin{array}{c}
v_{z}(x, y, 0)=0, \\
\tau_{x z}(x, y, 0)=0, \\
\tau_{y z}(x, y, 0)=0,
\end{array}\right\} \quad \text { in the } x y \text { plane, }
$$

A mesh with tetrahedral elements was chosen, with a higher density of elements close to the sphere, where larger gradients are expected. Convergence of the results was checked in two ways: by progressively enlarging the computational cell dimension and by refining the mesh at any fixed computation cell dimension. As an example, in Fig. 1 we show the sphere angular velocity versus the number of mesh elements, for a Maxwell fluid and the highest Deborah number investigated in this work $(\mathrm{De}=2.5)$, in a box with $L / R=14$. It can be concluded that a mesh size of 9000 elements suffices to obtain reliable results for the angular speed for this condition, whereas a deviation of about $13 \%$ is observed if the number of elements is decreased to 2500 .

The software used in this work, TFEM [Hulsen (2007)] discretizes the momentum equation using elastic viscous stress splitting (DEVSS-G) formulation [Guénette and Fortin (1995)] combined with the streamline upwind Petrov-Galerkin method [Brooks and Hughes (1982)] for the constitutive equation. Combined with the recently proposed log-conformation representation, this leads to a significant improvement of numerical stability [Fattal and Kupferman (2004); Hulsen et al. (2005)]. The numerical procedure is capable of transient calculations. The momentum and continuity equations are decoupled from the constitutive equation, and an implicit stress formulation is used. In this formulation the time-discretized constitutive equation is substituted into the momentum balance in order to obtain a Stokes like system. The torque-free condition [Eq. (3)] is imposed through constraints on the sphere surface, by means of Lagrange multipliers. In this way, the sphere rotation is automatically calculated by solving the augmented system of equa- 
tions. At each time step, the solution vector for $\boldsymbol{v}, p$, and $\boldsymbol{G}$ is computed, with $\boldsymbol{G}$ the "projected velocity gradient" [see Guénette and Fortin (1995); Bogaerds et al. (2002)]. Subsequently, the solution for the conformation vector $c$ is determined, through a combined Euler forward/backward formula of the constitutive equation with convection taken implicitly. A continuous quadratic interpolation for the velocity, a continuous linear interpolation for pressure, and a continuous bilinear interpolation for the stress tensor and auxiliary velocity gradient are used.

The time convergence has also been checked by decreasing the time step, $\Delta t$. Although the steady state solution is not affected too much if the time step is chosen large, the start-up behavior can substantially differ. In all simulations we set $\Delta t=0.0075$ assuring that no change in the transient trends occur if it is further decreased.

Finally, at each time step, we need to solve two linear nonsymmetric sparse systems. We use the parallel direct solver PARDISO [Schenk and Gartner (2004)].

The steady state results obtained with TFEM were checked for a few conditions against results obtained with a commercial software (Polyflow@), with which the hydrodynamic problem was iteratively solved directly at the steady state. The iterations were needed to determine the sphere angular velocity by a trial and error procedure. The iterative scheme to calculate the sphere angular velocity $\omega$ was built so as to attain zero torque on the sphere [Eq. (3)]. The iterations proceeded by successive corrections of the first guess for the unknown $\omega$ [Eq. (4)], and at each iteration step the entire flow problem was solved. For the viscoelastic constitutive equations in differential form we used a DEVSS/SU formulation with continuous quadratic interpolation for the velocity, continuous linear interpolation for pressure and continuous linear interpolation for the stress tensor and the projected velocity gradient components. Instead, a standard velocitypressure formulation is considered for the Bird-Carreau model (quadratic velocity and linear pressure). Upon discretization, the resulting fully coupled nonlinear system is linearized by the Newton-Rhapson method, and the sparse linear system is solved by a frontal direct method based on Gaussian elimination. The comparison between TFEM and Polyflow@ results was satisfactory in all the cases examined. In what follows, only TFEM results, which were computationally far more efficient, are presented and discussed.

\section{RESULTS}

In this section the results of our simulations for shear flow are presented. First, the steady state rotation of a sphere is investigated, and the effects of non-Newtonian behavior and viscoelasticity of the suspending fluid are analyzed. Then, the time evolution of the rotation rate is shown and its correlation with the building of the first normal stress difference of the suspending liquid is presented. All the simulations are performed with $L / R=14.0$, with $2 L$ the length of the cubic side of the computational domain. This dimension of the computation domain suffices to get invariant rotation rates (i.e., with relative errors below $10^{-3}$ ). The calculations were performed with 8000 elements.

\section{A. Steady state rotation of the sphere}

The rotation period of a rigid sphere immersed in a Newtonian fluid $\left(T_{\text {Newt }}\right)$ under shearing flow can be written in terms of a Jeffery period as $T_{\text {Newt }}=4 \pi / \dot{\gamma}$ [Einstein (1906)]. In what follows we use a normalized viscoelastic rotation period defined as $T$ $=T_{\mathrm{NN}} / T_{\text {Newt }}$, where $T_{\mathrm{NN}}$ is the rotation period of the sphere in the viscoelastic medium. Of course, for vanishing De, $T$ tends to unity. Experimental results [Astruc et al. (2003)] show that $T$ is an increasing function of the Deborah number, i.e., the sphere slows down 


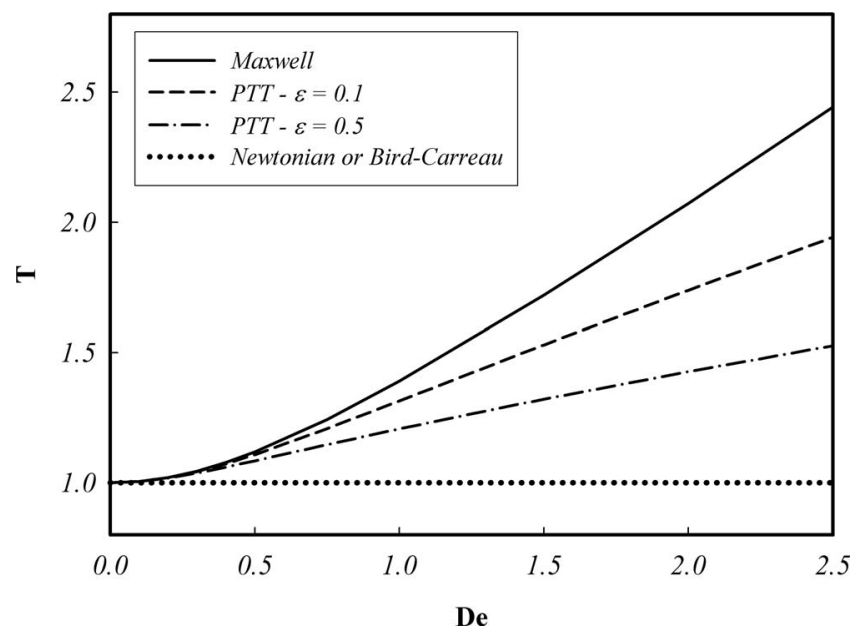

FIG. 2. Ratio of the rotation periods $T=T_{\mathrm{NN}} / T_{\mathrm{Newt}}$ as a function of De. Maxwell model (solid line), BirdCarreau model with two different values of the parameter $m$ (dotted line), PTT model with $\varepsilon=0.1$ (dashed line) and 0.5 (dot-dashed line).

by increasing De. Figure 2 shows $T$ plotted as a function of De varying in the range [0, 2.5] for the Bird-Carreau purely viscous fluid, the Maxwell fluid, and the PTT fluid.

The numerical results for the Bird-Carreau model are indicated with the dotted line. Two values of the constitutive parameter $m$ were considered $(m=2$ and $m=4)$, to investigate the possible effect of different shear thinning slopes [see Eq. (5)]. It turns out that the two curves exactly superimpose, and give $T=1$ throughout the explored Deborah range. Thus, a purely viscous, shear-thinning fluid as described with the Bird-Carreau model does not show any slowing down of the sphere, which keeps rotating as in the Newtonian case even at high De. Conversely, the Maxwell model (solid line in Fig. 2) apparently shows a substantial increase of $T$, i.e., a slowing down of the sphere angular velocity, with increasing De. The $T$ vs De curve reaches an asymptotic slope of $\sim 4 / 5$. Thus, fluid elasticity rather than shear thinning seems to be responsible for the slowing down of particle rotation at finite De. As it will be shown in the following, the Maxwell model, in fact, leads to the largest rotation period of the sphere, at least in the investigated De range.

Finally, the rotation period of the sphere in a PTT fluid [Eq. (7)] is also plotted in Fig. 2 , for two values of the constitutive parameter $\varepsilon$. The rotation period increases by increasing De, and, at a fixed De value the rotation period decreases with $\varepsilon$. The PTT curves lie in between the Maxwell and Bird-Carreau (=Newtonian) ones. In comparing PTT with Maxwell predictions, it so appears that a "weakening" of $N_{1}$ of the suspending liquid with increasing De (with respect to the Maxwell behavior $N_{1}=2 \mathrm{De}$ ) implies a smaller slowing down effect on the particle rotation. This aspect is further studied in Fig. 3 , where the dimensionless period ratio is plotted vs $\varepsilon$ at $\mathrm{De}=1.0$ and 2.0. The parameter $\varepsilon$ in the PTT model clearly modulates the relative slowing down of the sphere. The curve starts from the Maxwell prediction $(\varepsilon=0)$, and monotonically decreases.

The predictions with the Giesekus model with $\alpha=0.2$ and 0.4 are reported in Fig. 4, where also the Maxwell and Newtonian (or Bird-Carreau) results are plotted for the sake of comparison. As for the Maxwell model, an increase of the normalized period $T$ is observed with increasing De. As for the PTT model, such an increase is less steep than for the Maxwell fluid. 

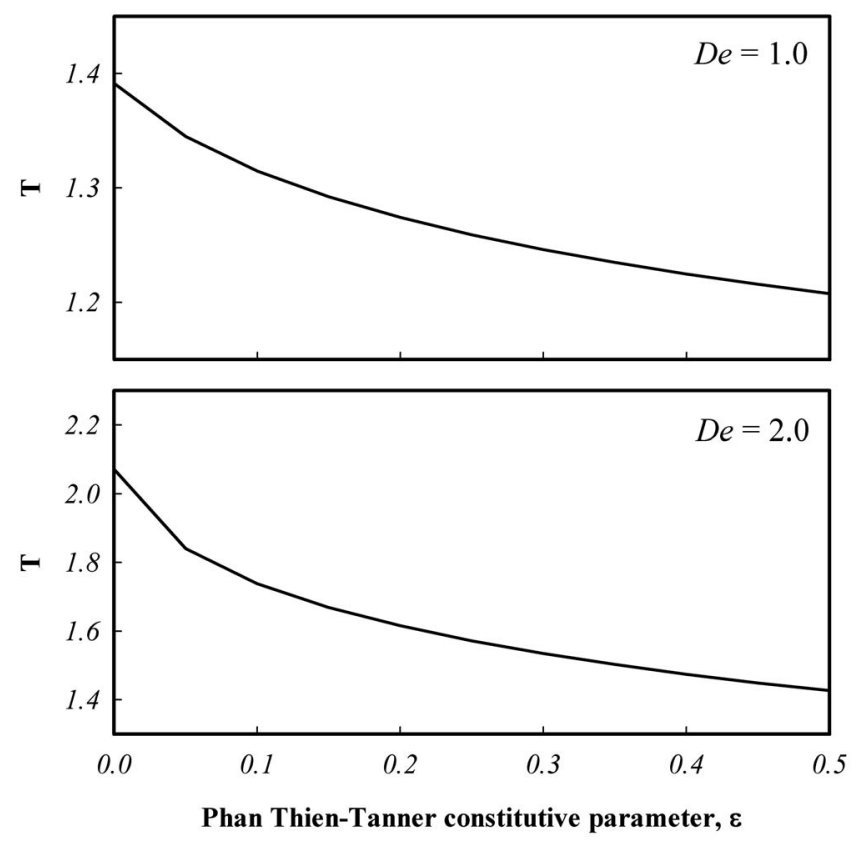

FIG. 3. Ratio of the rotation periods $T$ as a function of $\varepsilon$ for PTT model for De $=1.0$ and 2.0.

An interesting feature of the Giesekus fluid is the effect of the parameter $\alpha$ on the period ratio as can be deduced from Fig. 4. Indeed, at fixed De, a nonmonotonic behavior of $T$ with increasing $\alpha$ is found, as shown in Fig. 5 (contrast with Fig. 3 for the PTT model) for $\mathrm{De}=1.0$ and 2.0. The reason for such a nonmonotonic behavior is unclear. It should perhaps be considered that the Giesekus fluid is the only fluid investigated here

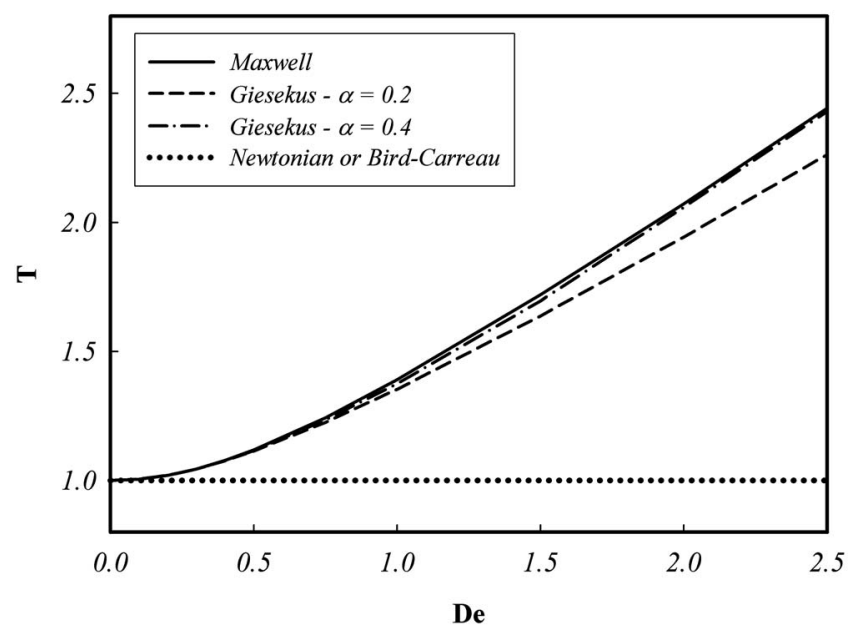

FIG. 4. Ratio of periods $T$ as a function of De. Maxwell model (solid line), Newtonian or Bird-Carreau (dotted line), and Giesekus model for $\alpha=0.2$ (dashed line) and $\alpha=0.4$ (dot-dashed line). 


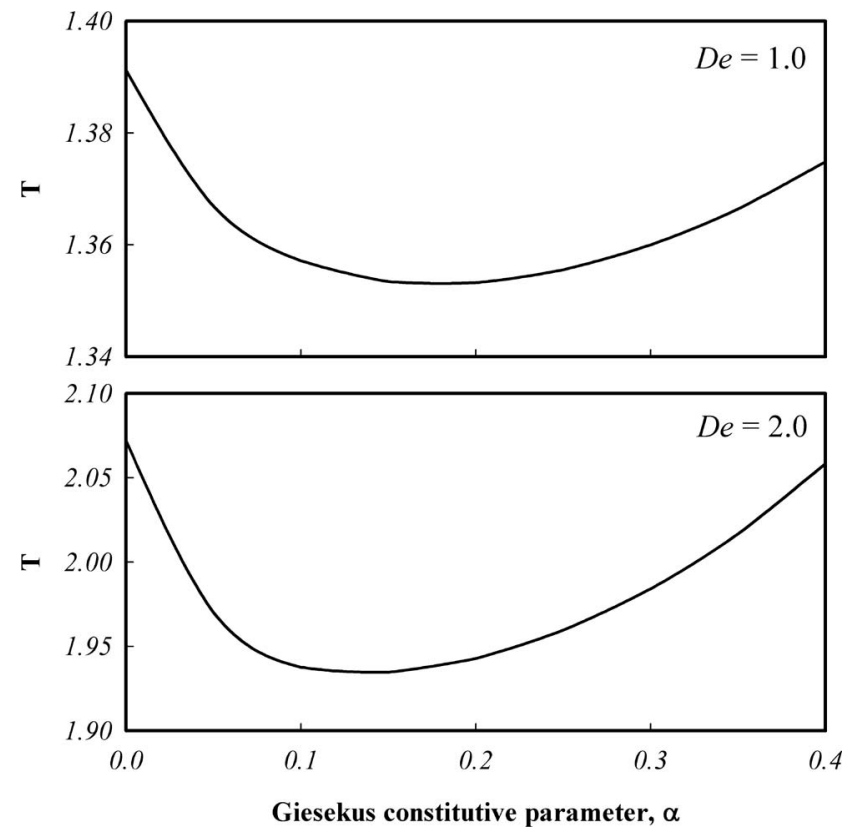

FIG. 5. Period ratio $T$ as a function of $\alpha$ for the Giesekus model at De $=1.0$ and 2.0.

possessing a nonzero second normal stress difference. The presence of a minimum for $T$ implies that for low values of $\alpha$ the rotation period departs from Maxwell prediction, to increase again for larger values of $\alpha$ (at a constant value of De).

\section{B. Transient results}

The disturbance velocity field around a particle is transient in nature and the viscoelastic fluids display transient effects in the shear and normal stresses. Therefore, it seems useful to compare the transient stress behavior of the neat suspending liquid during shear start up versus the transient behavior of the rotation period of the sphere, when immersed in that same liquid, at different De values. The nondimensional viscosity, first normal stress difference and rotation period will be plotted versus strain for the Maxwell, PTT, and Giesekus models.

Figure 6 shows the predictions for the Maxwell fluid. The steady state values are always attained monotonically. As expected, the normal stress differences take about twice as long to achieve steady state compared to the shear stress. The rotation rate reaches its steady state value on a time scale similar to that of the first normal stress difference rather than that of the viscosity.

Figure 7 shows the predictions for the PTT model with $\varepsilon=0.5$. This fluid shows the appearance of a slight overshoot in the stress responses at high De values. (Thinning of both viscosity and $N_{1}$ is observed in the investigated De range.) At the highest De value, also the rotation rate of the sphere is clearly seen to approach the steady state in a nonmonotonic way. Interestingly, the overshoot in $T$ occurs at a strain quite close to that of the $N_{1}$ overshoot.

Finally, Fig. 8 shows the predictions for the Giesekus model with $\alpha=0.4$. The same kind of behavior found for the PTT model is observed in this case. Again, the overshoot 

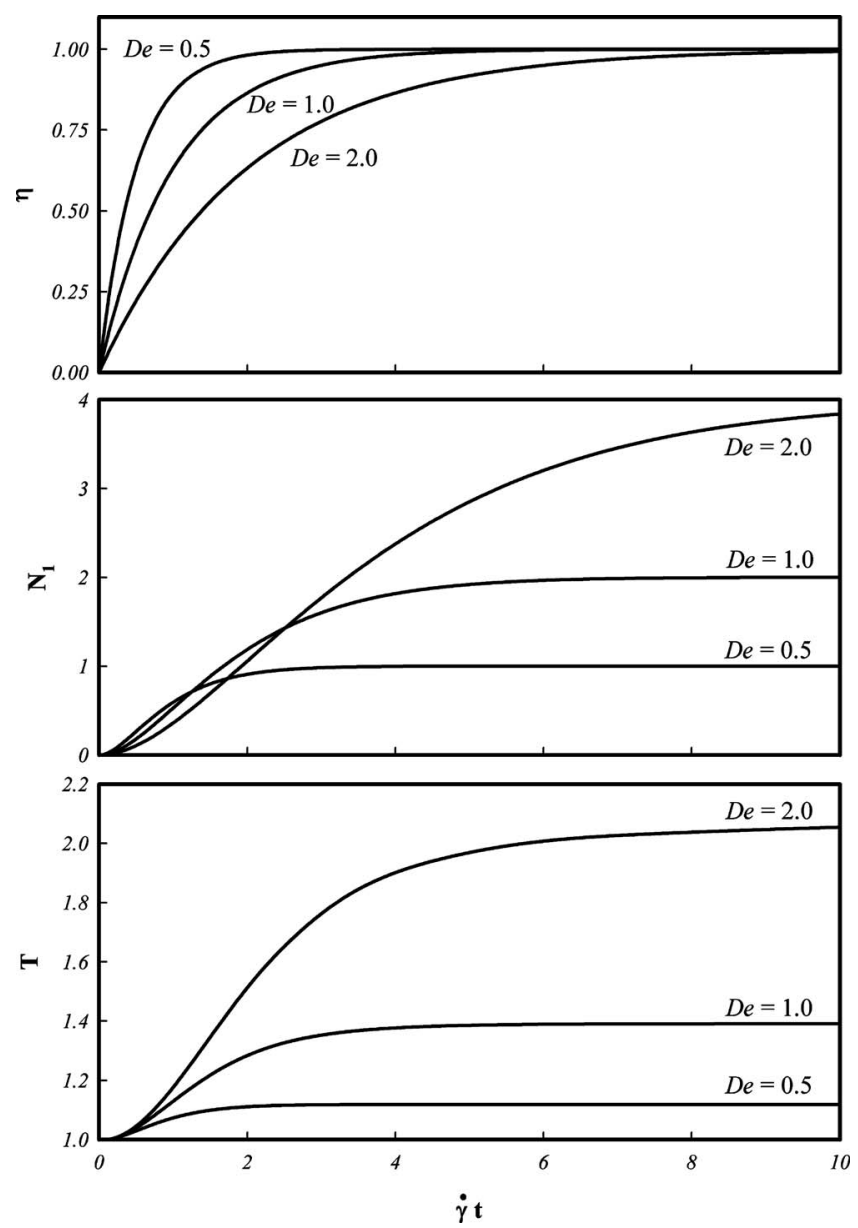

FIG. 6. Nondimensional viscosity and first normal stress difference of the neat Maxwell model, and the nondimensional rotation period of the sphere immersed in the Maxwell fluid at various Deborah numbers plotted versus strain.

positions in the $T$ plot closely correspond to those observed in the first normal stress difference of the neat Giesekus fluid, rather than with those of the viscosity.

In all three cases, time effects in the rotation rate dynamics follow the time evolution of the normal stresses of the suspending liquid. Thus, also the transient results confirm the substantial effect of normal stresses on the rotational behavior of the spheres, already found in the steady state case. This might seem somewhat counterintuitive as, at first sight, the time effects in the shear stress should be of importance. However, if the only changes with respect to the Newtonian case were a slowing down of the shear stress buildup and decay, this would not lead to a significant change in the torque acting over the particle surface. The point at which the tangential stresses start to exert a torque would be "delayed," but also the point where the stresses significantly decay would shift further on the particle surface. Hence, the same nature of the streamlines around the sphere is roughly conserved, they only become slightly tilted. On the other hand, normal stresses strongly influence the pressure and velocity fields around the particle, inducing a symmetry breaking with respect to the Newtonian case (see the next section for a detailed analysis of the streamlines around the sphere). Consequently, the normal stress effects 

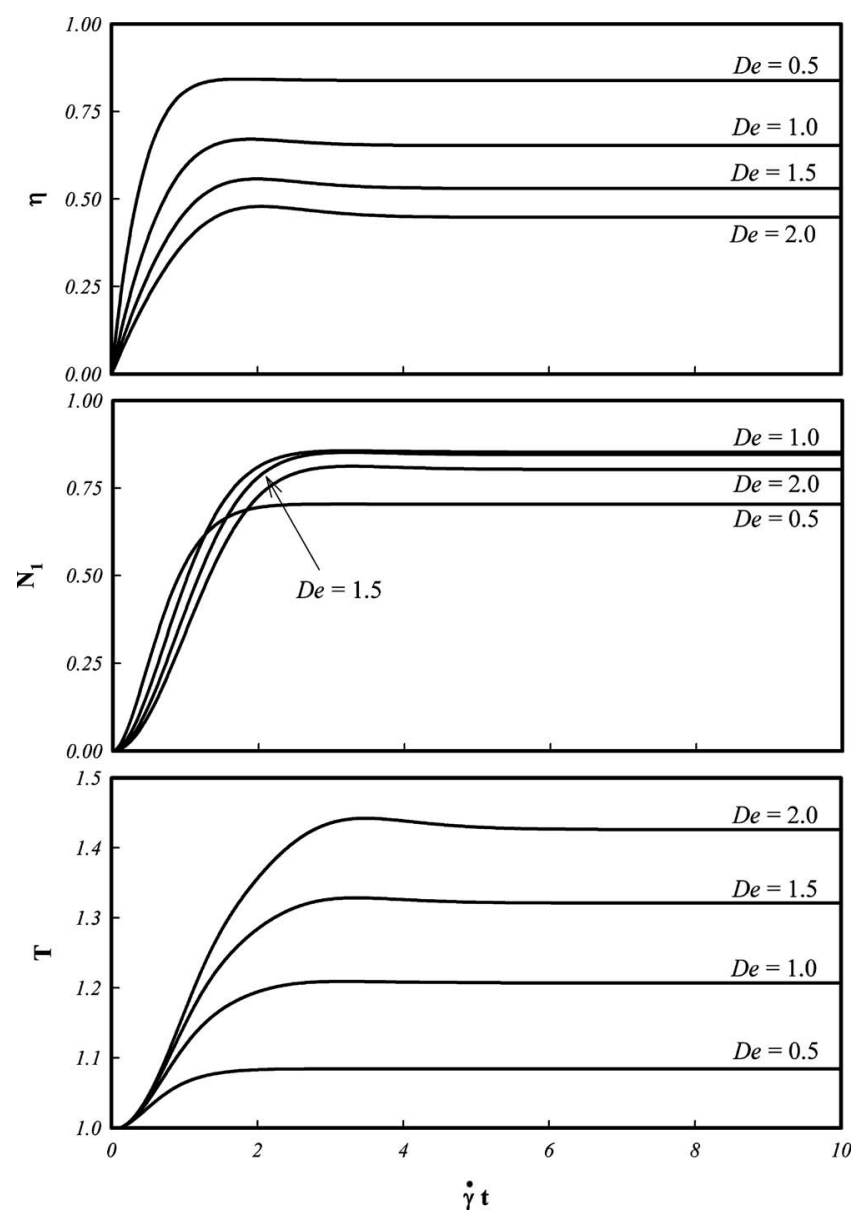

FIG. 7. Nondimensional viscosity and first normal stress difference of the neat PTT model for $\varepsilon=0.5$ and the nondimensional rotation period of the sphere immersed in the PTT fluid at various Deborah numbers plotted versus strain.

will profoundly change the streamlines, the local velocities, and the deformation rates close to the particle surface, bearing some similarity with the effects of fluid inertia on particle motion [Mikulencak and Morris (2004)]. To assess the relevance of the normal stress effects, a quantitative comparison with experimental data on particle rotation speeds in different viscoelastic fluids is needed.

\section{Streamlines}

In the previous sections, we show that the viscoelasticity of the suspending fluid profoundly alters the rotation rate of a suspended particle in shear flow. Of course, the flow field surrounding the particle will be modified as well. To give an idea about the changes in the flow structure due to the non-Newtonian character of the fluid and to show the differences from the Newtonian case, in this section the streamlines for a Newtonian as well as a viscoelastic suspending liquid are presented. We limit our observations to the $x y$ plane. Due to the mirror symmetry at the $x y$ plane, a streamline starting from a point on such a plane remains on that same plane, so it can be easily observed through a 2D plot. 

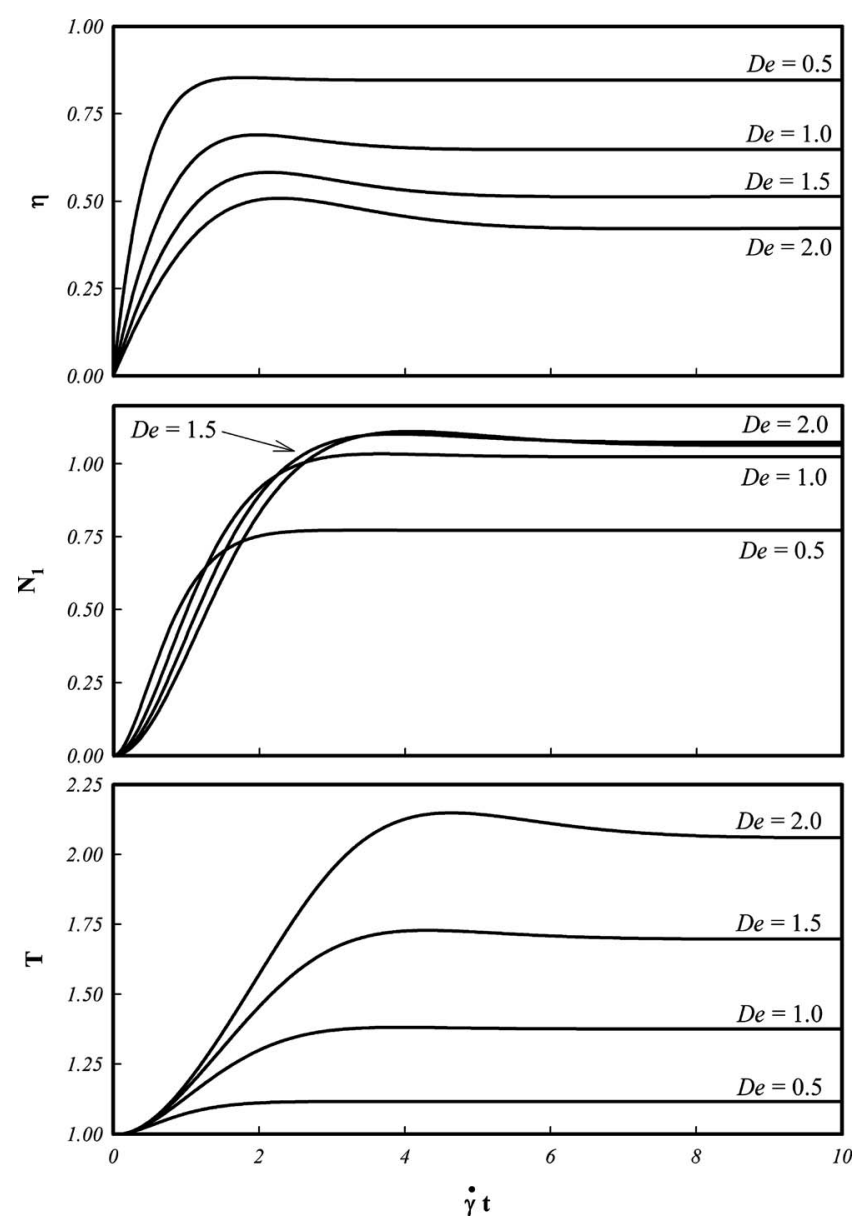

FIG. 8. Nondimensional viscosity and first normal stress difference of the neat Giesekus model for $\alpha=0.4$ and the nondimensional rotation period of the sphere immersed in the Giesekus fluid at various Deborah numbers plotted versus strain.

The streamlines can be obtained in general by integrating the following kinematic equations:

$$
\begin{aligned}
& \dot{x}=v_{x}(x, y, z), \quad x(0)=x_{0}, \\
& \dot{y}=v_{y}(x, y, z), \quad y(0)=y_{0}, \\
& \dot{z}=v_{z}(x, y, z), \quad z(0)=z_{0},
\end{aligned}
$$

where the right hand side is given by the solution of the full $3 \mathrm{D}$ problem and $\left(x_{0}, y_{0}, z_{0}\right)$ is a starting point. Of course, only the first two equations of Eqs. (11) are needed in the $x y$ plane, $v_{z}$ being 0 . Since we solve on one quarter of the domain $(y>0, z>0)$, the velocity field in the plane for $y<0$ is obtained by exploiting the antisymmetry with respect to the sphere center

$$
v_{x}(x, y, 0)=-v_{x}(-x,-y, 0),
$$




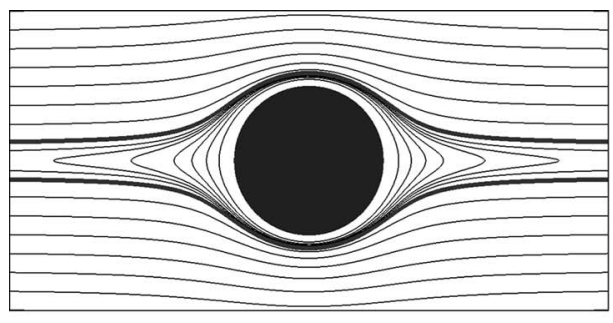

a) Newtonian

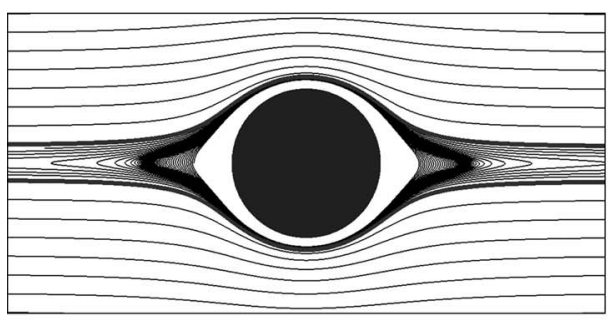

c) Giesekus $-\alpha=0.2-D e=0.3$

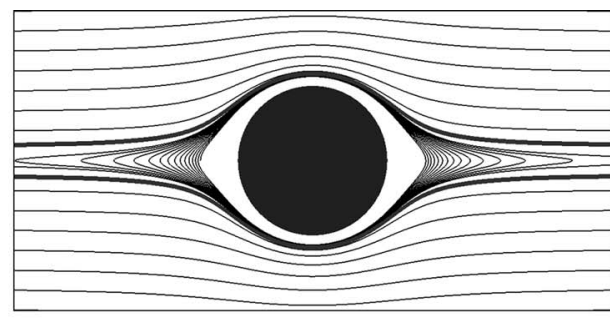

b) Giesekus $-\alpha=0.2-D e=0.1$

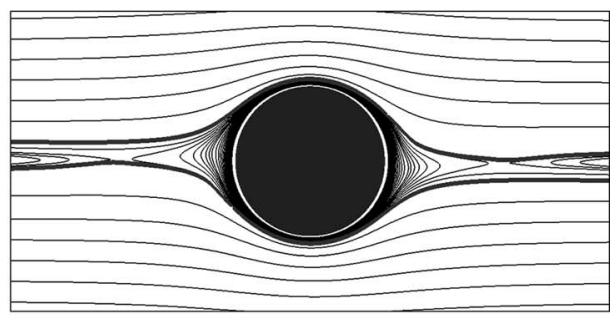

d) Giesekus $-\alpha=0.2-D e=1.0$

FIG. 9. Streamlines on the $x y$ plane for Newtonian and viscoelastic case (Giesekus suspending fluid with $\alpha$ $=0.2$ and three different Deborah numbers).

$$
v_{y}(x, y, 0)=-v_{y}(-x,-y, 0) .
$$

Finally, an adaptive Runge-Kutta algorithm is used in order to integrate the Eqs. (11).

As is well known, for a single inertialess sphere in an unbounded Newtonian fluid subjected to shear flows we can divide the $x y$ plane in two zones characterized by a different behavior of the streamlines. In the "inner" one, the streamlines are given by an infinite set of closed lines surrounding the sphere. Such a zone extends to infinity and becomes thinner and thinner far from the sphere. In the external region, instead, a fluid particle coming from $( \pm)$ infinity moves to $(\mp)$ infinity, according to a distorted path if it is sufficiently close to the particle. The just described situation is depicted in Fig. 9(a). The inner and the outer regions are separated by the grey bold lines. Notice that for a Stokes flow, a fully symmetric scenario (i.e., fore-aft and up-down) is observed.

In Fig. 9(b), a viscoelastic suspending fluid is considered. The fluid is modeled with a Giesekus model $(\alpha=0.2)$ and the Deborah number is 0.1 . In spite of the very low De value, a qualitative difference with respect to the previous case appears. In the inner region (enclosed by the grey bold lines) the streamlines open and move far from the sphere as time goes on [notice that within the grey separatrices only one orbit is depicted starting from $(-1.5,0)]$. Such a situation, of course, leads to a symmetry breaking along the $x$ and $y$ directions, maintaining of course the antisymmetry with respect to the origin. The result just presented is in qualitative agreement with the analytical solution of Subramanian and Koch (2007) for a single, inertialess sphere in a SOF.

By further increasing the Deborah number [see Fig. 9(c)], the inner zone is divided into two subregions. Starting close to the sphere, the streamlines tend to the distorted closed black curve reported in the figure. A similar trend is found by starting outside such a curve, but still within the grey bold lines delimiting the inner region. To better show such a situation, we report in Fig. 10 an inset of Fig. 9(c). The starting points are labeled as " 1 " $(-1.5,0)$ and " 2 " $(-3.5,0.2)$. Notice that only two trajectories are reported within the inner region and both tend to the black curve. 


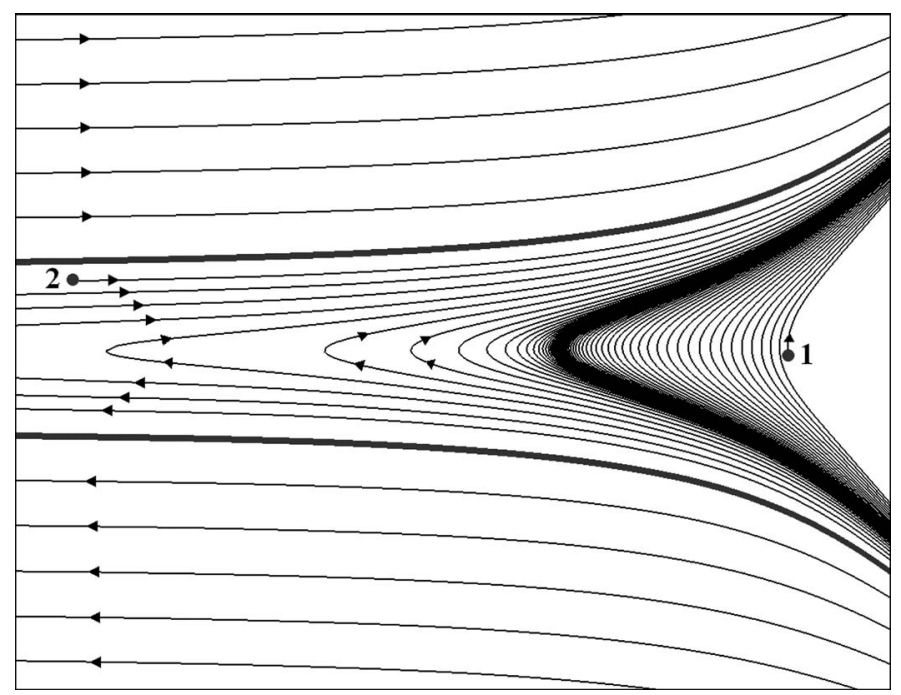

FIG. 10. Zoom of the streamlines on the $x y$ plane for a viscoelastic case (Giesekus suspending fluid with $\alpha$ $=0.2$ and $\mathrm{De}=0.3$ ).

As De increases, we found that the "attractive" distorted orbit approaches more and more the sphere. In Fig. 9(d) the case $\mathrm{De}=1.0$ is reported. The orbit is very close to the sphere. Furthermore, two recirculation zones appear. A fluid particle within these zones never crosses the solid particle but goes back to infinity inverting its motion. The existence of the recirculation zones is better shown by looking at the inset of Fig. 9(d), reported in Fig. 11.

The arising of two saddle points ("S" and its antisymmetric) is also evident. Notice that the trajectory starting from point 1 approaches the sphere and tends towards the black orbit close to the sphere. In conclusion, the inner region is now divided into three sub-

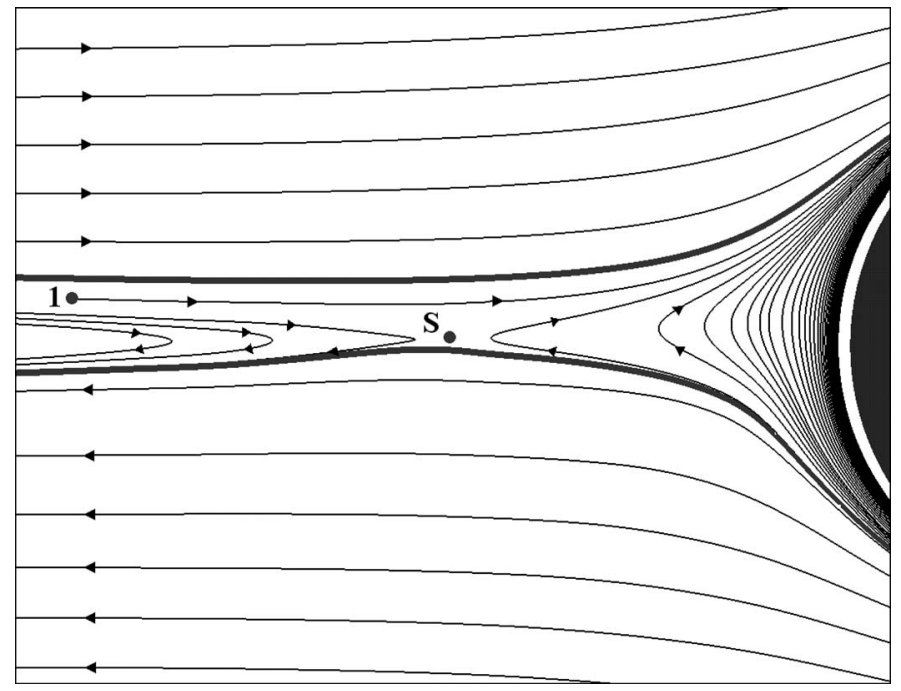

FIG. 11. Zoom of the streamlines on the $x y$ plane for a viscoelastic case (Giesekus suspending fluid with $\alpha$ $=0.2$ and $\mathrm{De}=1.0$ ). 
regions: the recirculation zone, the set of trajectories starting in between the recirculation region and the black orbit and the set of trajectories in between the sphere and the black orbit. The latter two tend towards the black orbit itself.

By increasing the Deborah number up to 2.5 (the maximum investigated in this work), the scenario is found to be the same, with the two recirculation regions becoming larger and moving towards the sphere.

\section{CONCLUSIONS}

In the present work we have used numerical methods to predict the effect of viscoelasticity on the rotation of inertialess, density matched, rigid, non-Brownian spheres in suspension in an externally imposed steady shear flow. Four different constitutive equations were considered to model the rheology of the suspending liquid, namely the BirdCarreau, Maxwell, PTT, and Giesekus models. The fully 3D nature of the problem, plus the nonzero De values examined required numerical simulations of the hydrodynamics. The finite element simulations were performed with the TFEM code, and spot calculations (at the steady state) were performed with Polyflow@ to ascertain the validity of the obtained results up to $\mathrm{De}=2.5$.

It was observed that the viscoelasticity of the suspending liquid leads to a substantial slowing down of the angular velocity of the sphere with respect to the Newtonian case. This prediction is in qualitative agreement with the limited available experimental data. It was also shown that different viscoelastic liquids imply different intensities of slowing down, an outcome for which no experimental evidence is as yet available. It is fair to note, however, that we did not reach any simple intuitive explanation of the rotational slowing effect, even though a rather strict correlation with normal stresses of the suspending liquid is observed in the transients.

Finally, to highlight the changes in the flow field due to the viscoelasticity of the suspending fluid, the streamlines at different Deborah number are analyzed. In agreement with the analytical theory for a SOF, at very low De values, the trajectories surrounding the particle open up and move far from the sphere, contrary to the Newtonian case that is characterized by closed orbits. By increasing De, an attractive closed orbit around the sphere appears. Such an orbit approaches the particle as De increases. At significantly high De values, two recirculation regions arise: a fluid particle inside these regions never cross the sphere but inverts its motion coming back to infinity.

The predictions reported here will be compared with experimental data of spheres in selected viscoelastic media in a forthcoming paper.

\section{ACKNOWLEDGMENTS}

FS acknowledges a graduate fellowship of the Fund for Scientifc Research-Flanders (FWO-Vlaanderen). This work was partially funded by FIRB Mapionano and PrismaINSTM 2008.

\section{References}

Astruc, M., S. Vervoort, H. O. Nouatin, T. Coupez, Y. De Puydt, P. Navard, and E. Peuvrel-Disdier, "Experimental and numerical study of the rotation and the erosion of fillers suspended in viscoelastic fluids under simple shear flow," Rheol. Acta 42, 421-431 (2003).

Bogaerds, A. C. B., A. M. Grillet, G. W. M. Peters, and F. P. T. Baaijens, "Stability analysis of polymer shear 
flows using the extended pom-pom constitutive equations," J. Non-Newtonian Fluid Mech. 108, 187-208 (2002).

Brooks, A. N., and T. J. R. Hughes, "Streamline upwind/Petrov-Galerkin formulations for convection dominated flows with particular emphasis on the incompressible Navier-Stokes equations," Comput. Methods Appl. Mech. Eng. 32, 199-259 (1982).

Brunn, P. O., "Slow motion of a sphere in a second-order fluid," Rheol. Acta 15, 163-171 (1976).

Einstein, A., "Eine Neue Bestimmung der Molejul Dimensionen,” Ann. Phys. 19, 289-306 (1906).

Fattal, R., and R. Kupferman, "Constitutive laws for the matrix-logarithm of the conformation tensor," J. Non-Newtonian Fluid Mech. 123, 281-285 (2004).

Gauthier, F., H. L. Goldsmith, and S. G. Mason, "Particle motions in non-Newtonian media. I. Couette flow," Rheol. Acta 10, 344-364 (1971).

Guénette, R., and M. Fortin, "A new mixed finite element method for computing viscoelastic flows," J. NonNewtonian Fluid Mech. 60, 27-52 (1995).

Hulsen, M. A., TFEM: A Toolkit for the Finite Element Method, Userguide, 2007.

Hulsen, M. A., R. Fattal, and R. Kupferman, "Flow of viscoelastic fluids past a cylinder at high Weissenberg number: Stabilized simulations using matrix logarithms," J. Non-Newtonian Fluid Mech. 127, 27-39 (2005).

Hwang, W. R., M. A. Hulsen, and H. E. H. Meijer, "Direct simulations of particle suspensions in a viscoelastic fluid in sliding bi-periodic frames," J. Non-Newtonian Fluid Mech. 121, 15-33 (2004).

Larson, R. G., Constitutive Equations for Polymer Melts and Solutions (Butterworth-Heinemann, London, 1988).

Leal, L. G., "The motion of small particles in nonNewtonian fluids," J. Non-Newtonian Fluid Mech. 5, 33-78 (1979).

Mikulencak, D. R., and J. F. Morris, "Stationary shear flow around fixed and free bodies at finite Reynolds number,” J. Fluid Mech. 520, 215-242 (2004).

Schenk, O., and K. Gartner, "Solving unsymmetric sparse systems of linear equations with PARDISO," FGCS, Future Gener. Comput. Syst. 20, 475-487 (2004).

Subramanian, G., and D. L. Koch, "Heat transfer from a neutrally buoyant sphere in a second-order fluid," J. Non-Newtonian Fluid Mech. 144, 49-57 (2007).

Truesdell, C. A., and W. Noll, in Encyclopaedia of Physics, edited by S. Flugge (Springer, Berlin, 1965), Vol. III/3. 